\title{
Angiogenesis in Glioblastoma
}

\author{
Sunit Das, M.D., Ph.D. and Philip A. Marsden, M.D. \\ Keenan Research Centre of the Li Ka Shing Knowledge Institute and the Departments of \\ Neurosurgery and Medicine, St. Michael's Hospital (S.D.), and the University of Toronto (P.A.M.) \\ - both in Toronto.
}

Glioblastoma multiforme is the most common malignant primary brain tumor in adults. Mean progression-free survival is just over 6 months; treatment with surgical resection, chemotherapy, and radiation is invariably followed by tumor recurrence. Aggressive surgical resection decreases the tumor-cell burden by $99 \%$, leaving about 100 million cells; cytotoxic adjuvant therapy with an agent such as temozolomide reduces the burden to 10 million cells. The mean overall survival achieved with repeat surgery or irradiation or with salvage chemotherapy remains only 14.6 months.

What accounts for this poor prognosis? Examination of the surrounding, otherwise normal brain tissue at diagnosis uniformly reveals the presence of infiltrative glioma cells. Moreover, glioma cells that are resistant to the effects of chemotherapy and radiation account for tumor progression. Recent developments have focused on two seemingly unrelated concepts. First, interactions between tumor cells and blood vessels seem to facilitate tumor growth. Indeed, the angiogenesis inhibitor bevacizumab has been shown to increase progression-free survival in patients with newly diagnosed or recurrent glioblastoma multiforme, presumably by inhibiting both the formation of vessels dependent on vascular endothelial growth factor (VEGF) and vascular permeability in these highly vascular tumors. Second, many cancers, including glioblastoma multiforme, have been found to be driven by a subpopulation of cancer stem cells. Several attributes distinguish glioma stem cells from other glioma cells, including the capacity for self-renewal and the generation of tumors after xenotransplantation. Glioma stem cells possess properties that make them resistant to radiation and chemotherapy, and they may serve as a repository for glioblastoma multiforme, allowing its recurrence. ${ }^{1}$ Several recent studies wed these concepts.

One pathologic feature of glioblastoma multiforme that distinguishes it from lower-grade glial tumors is microvascular proliferation. Glioblastoma multiforme is highly vascular. Several mechanisms have been implicated in the robust formation of blood vessels observed in these tumors (Fig. 1). One is the sprouting of capillaries from preexisting blood vessels by means of endothelial proliferation, which seems to depend on the presence of hypoxia in the tumor core. Indeed, hypoxia-inducible factor, which enhances the transcription of $V E G F$, is activated in glioblastoma multiforme. Another mechanism of new blood-vessel formation is 
the release of angiogenic factors from the tumor cells. These factors recruit cells that may participate in vessel formation.

The work of four independent groups has revealed that glioma stem cells may contribute to blood-vessel formation in tumors by differentiating into endothelial cells or pericytes, a phenomenon that is perhaps best termed vascular mimicry. ${ }^{2-4}$ This work is important because it indicates that the progeny of glioma stem cells are not limited to a neuroepithelial cell lineage. Using human surgical specimens, Wang et al. ${ }^{2}$ and Ricci-Vitiani et al. ${ }^{3}$ found a subset of endothelial cells lining tumor vessels that carried the same genetic abnormalities (e.g., amplification of epidermal growth factor receptor [EGFR] or mutation of TP53) that mark adjacent malignant tumor cells. Using acutely dissociated tumor cells, the two research groups also revealed the presence of a population of neoplastic endothelial progenitor cells in vivo, and they showed that a subpopulation of glioma stem cells gave rise to endothelial cells in vitro. These cells, when intracranially transplanted into immuno-compromised mice, gave rise to tumors in which a proportion of the endothelial cells contributing to blood vessels originated from the transplanted human glioma cells rather than from the mice. Using a mouse model of glioblastoma multiforme, Soda et al. ${ }^{4}$ observed a subpopulation of endothelial cells within these tumors that expressed both endothelial-cell-specific and tumorspecific antigens. All three groups observed that tumor-derived endothelial cells are more prevalent within the core of the glioblastoma multiforme and are less frequently found in the tumor periphery.

A recent report by Cheng et al. ${ }^{5}$ provides strong evidence that glioma stem cells also generate the vascular pericytes that surround blood vessels within glioblastoma multiforme. The investigators observed that glioma stem cells are able to form cells of the vascular pericyte lineage in vitro and showed that glioma stem cells can give rise to pericytes in a mouse xenograft model in vivo. Furthermore, they found that the majority of tumor pericytes carried the same genetic alterations found in the glioblastoma multiforme. Surprisingly, neoplastic pericytes were also found in the peritumoral normal brain. These findings have important implications for glioblastoma therapy: increased pericyte coverage is thought to confer resistance to bevacizumab and may be a consequence of the profound hypoxia that accompanies VEGF blocking.

These findings have provided us with a more comprehensive understanding of how blood vessels form within glioblastoma multiforme. In these tumors, the blood vessels themselves are, at least in part, neoplastic. The extent to which vascular mimicry in glioblastoma multiforme preferentially results in the generation of endothelial cells derived from glioma stem cells rather than pericytes remains to be defined. Other key questions also arise. What is the functional significance of vascular mimicry in glioblastoma multiforme with respect to the niche microenvironment of the glioma stem cell? Is this phenomenon common to all tumors of the glioblastoma multiforme type, or does it define a glioblastoma multiforme subtype? Finally, how do tumor vessels derived by means of vascular mimicry differ from those derived from their non-neoplastic counterparts? These questions notwithstanding, the collective work of these researchers points to the use of stem-cell-driven angiogenesis as a target for novel, experimental approaches to the treatment of glioblastoma multiforme. 
Indeed, the disruption of vascular mimicry, either through the specific targeting of tumorderived endothelial cells ${ }^{2,3}$ or through pericytes, ${ }^{5}$ slowed tumor growth.

\section{References}

1. Chen J, Li Y, Yu TS, et al. A restricted cell population propagates glioblastoma growth after chemotherapy. Nature. 2012; 488:522-6. [PubMed: 22854781]

2. Wang R, Chadalavada K, Wilshire J, et al. Glioblastoma stem-like cells give rise to tumour endothelium. Nature. 2010; 468:829-33. [PubMed: 21102433]

3. Ricci-Vitiani L, Pallini R, Biffoni M, et al. Tumour vascularization via endothelial differentiation of glioblastoma stem-like cells. Nature. 2010; 468:824-8. [PubMed: 21102434] [Errata, Nature 2011;469:432, 477:238.]

4. Soda Y, Marumoto T, Friedmann-Morvinski D, et al. Trans-differentiation of glioblastoma cells into vascular endothelial cells. Proc Natl Acad Sci U S A. 2011; 108:4274-80. [PubMed: 21262804]

5. Cheng L, Huang Z, Zhou W, et al. Glioblastoma stem cells generate vascular pericytes to support vessel function and tumor growth. Cell. 2013; 153:139-52. [PubMed: 23540695] 


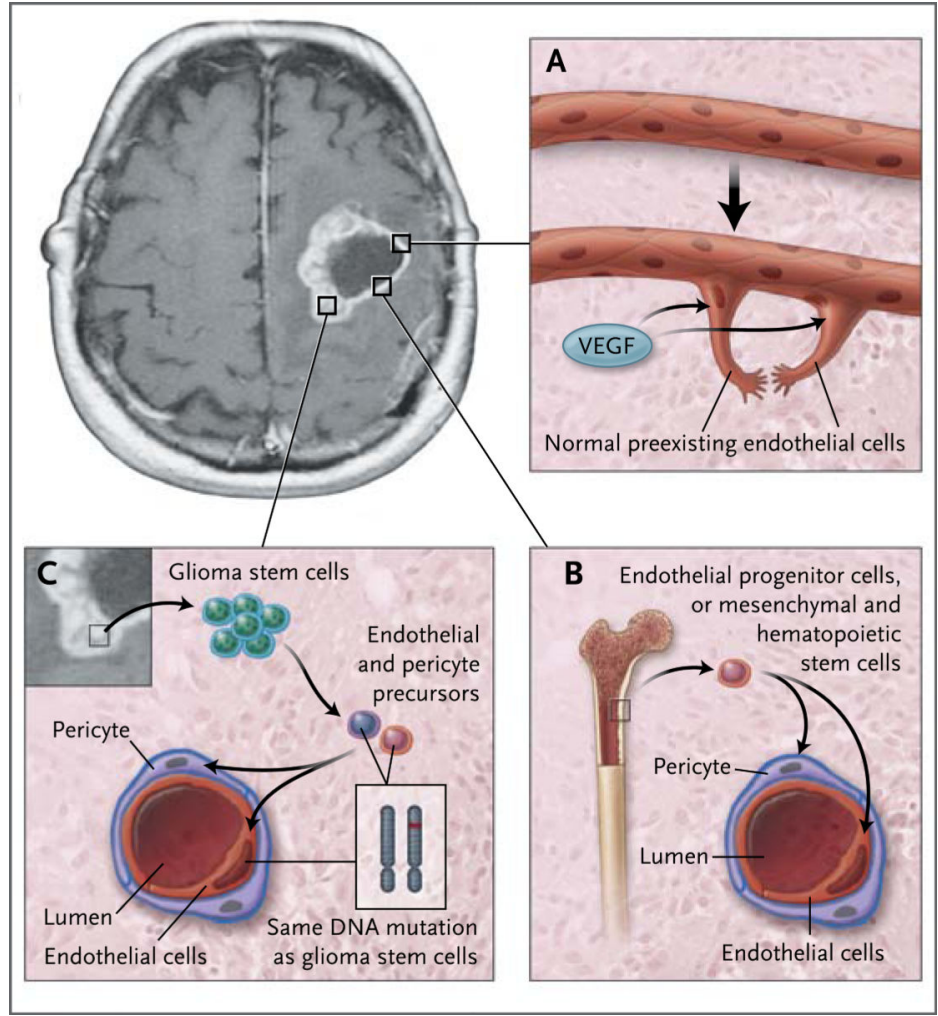

Figure 1. Angiogenesis in Glioblastoma Multiforme

A variety of pathways have been described for the formation of new blood vessels in glioblastoma multiforme. Hypoxic tumor cells, especially those surrounding the necrotic core, release vascular growth factors, such as vascular endothelial growth factor (VEGF), that stimulate the formation of new blood vessels from preexisting normal endothelial cells (Panel A). This process involves both endothelial-cell proliferation and enhanced vascular permeability. New blood-vessel formation also occurs through the recruitment of bone marrow-derived endothelial progenitor cells or through mesenchymal or hematopoietic stem cells (Panel B). These cells migrate from the systemic circulation into the tumors. Recent work shows that glioma stem cells contribute to the formation of tumor blood vessels by differentiating into endothelial cells or pericytes, contractile cells that wrap around the endothelial cells of small blood vessels (Panel C). In the normal brain, the neurovascular unit is composed of neurons, astrocytes, endothelial cells, and pericytes. Pericytes play a critical role in the regulation of blood-vessel function: they control local blood flow and the permeability of the blood-brain barrier, and they communicate directly with endothelial cells through gap junctions and soluble paracrine signals. 